\title{
LA PREPARACIÓN DE LOS PROFESIONALES EN LOS CENTROS UNIVERSITARIOS MUNICIPALES Retos y perspectivas
}

The Preparation of Professionals in Municipal University Centers: Challenges and Prospects

\author{
DABerquis ISABel Rodríguez ReYes
}

Universidad de Cienfuegos "Carlos Rafael Rodríguez", Cuba

\section{KEY WORDS}

Age Group

Heterogeneity

Adult Pedagogy

Universalization of Higher

Education

\section{ABSTRACT}

The process of universalization of Higher Education in Cuba, together with the implementation of novel and varied curriculums that contribute to increase and diversify the ways of accessing to university studies, have made possible the integration of several age groups and diverse previous preparation of the students enrolled in the different courses given at the municipal campuses. Thus, there has also been an increase of the teaching staff made up by professionals arriving from other educational levels and/or professions who require an up-dated continuing professional development on pedagogical, methodological and didactical topics.

\section{PALABRAS CLAVE}

\section{Etárea}

Heterogeneidad

Andragogía

Universalización de la

Educación Superior

\section{RESUMEN}

El proceso de universalización de la Educación Superior en Cuba y, la implementación de novedosos y variados programas educativos que incrementan $y$ diversifican sus fuentes de ingreso, han propiciado la heterogeneidad etárea y de conocimientos en la composición de las matrículas de los distintos centros universitarios de los territorios. Unido a esto, el necesario incremento del claustro con profesionales procedentes de disímiles sectores de la sociedad, exigen una adecuación en el accionar pedagógico, metodológico y didáctico de estos docentes. 


\section{Introducción}

$\mathrm{L}$ a educación es un derecho humano fundamental y como tal, es un elemento clave del desarrollo sostenible, de la paz y la estabilidad en cada país y entre las naciones, $y$, por consiguiente, un medio indispensable para participar en los sistemas sociales y económicos del siglo XXI.

En consecuencia, el perfeccionamiento de la enseñanza se ha convertido, en las últimas décadas, y en casi todos los países, en centro de atención de investigadores, didactas y pedagogos. Ello es el resultado del acelerado desarrollo científico y tecnológico, que engendra nuevas y elevadas exigencias a la escuela contemporánea y más específica a la formación inicial y permanente que se desarrolla en los centros de Educación Superior.

En Cuba, país en vías de desarrollo, cuyo pueblo se halla enfrascado desde hace más de 58 años en la realización de una revolución social en condiciones excepcionales, la necesidad de un mejoramiento sustancial en la formación académica y especializada de sus ciudadanos, adquiere dimensiones extraordinarias. Por tal motivo, el objetivo de este trabajo es la preparación pedagógica del profesional que se desempeña como profesor en los centros universitarios municipales para elevar la calidad de los procesos formativos.

Las actuales condiciones de la nueva universidad cubana exige la ampliación de un claustro universitario con profesionales del territorio que se desempeñan en disímiles sectores de la sociedad y que, en la mayoría de los casos, no han recibido ningún tipo de formación pedagógica. Tal planteamiento, unido a la masividad y heterogeneidad etárea, social, cultural y académica que experimentan las matrículas en este nivel de enseñanza, son fundamentos que exigen una reconceptualización en el accionar pedagógico de dichos profesionales.

En el presente trabajo, se aclaran conceptos pedagógicos y andragógicos, se aborda el perfil y las características deseables del profesor de la actual universidad cubana, los ejes fundamentales que sustentan el proceso docente educativo y el diagnóstico como uno de los aspectos esenciales que debe tener en cuenta este docente para el desempeño de sus funciones.

\section{Desarrollo}

\section{Concepciones pedagógicas y andragógicos}

La importancia estratégica del factor humano en nuestra sociedad, la necesidad de responder o adelantarse a los cambios constantes y acelerados, en el afán de construir una sociedad más justa en un mundo unipolar, nos obligan a revisar y readecuar el modo de entender, valorar, planificar y ejecutar nuestras acciones relacionadas con la educación y más aún cuando se trata de cambios tan necesarios como el que nos impone la actual Revolución Educacional cubana.

Con tal afán surge el presente trabajo, pues el sentido común pareciera indicarnos que basta con un buen nivel de conocimiento sobre el contenido que imparte, para ser un agente eficaz en la labor que realiza, sin embargo, al observar con detención el desempeño real de los profesionales que ejercen como profesores en el proceso de universalización, constatamos que ponen en práctica un modelo tradicional, el que conocen, $y$ al que ellos mismos han sido sometidos desde niños: enseñan utilizando un modelo pedagógico tradicional, concebido y diseñado para el aprendizaje de niños y adolescentes.

El gran inconveniente es que muchas de estas metodologías pueden ser contraproducentes al ser aplicadas en el contexto actual de las matrículas de la Educación Superior, ya que por su carácter heterogéneo en cuanto a edades, conocimientos y estrategias de aprendizaje, la situación y relación con el entorno, de estos educandos, es radicalmente diferente a la de un niño o un adolescente.

De otro lado, el desarrollo de la psicología del aprendizaje y de la pedagogía en las últimas décadas ha confirmado ampliamente los cuestionamientos que desde tiempo atrás formulaban muchos educadores y psicólogos al enfoque tradicional de la enseñanza, centrado en los contenidos de información y no en las necesidades y los procesos personales y grupales de los estudiantes

Teniendo en consideración que las matrículas de los centros universitarios de los territorios están compuestas por personas jóvenes y adultas, se hace necesario retomar conceptos pedagógicos y andragógicos que optimicen el proceso docente educativo con dichas matrículas. 
Hoy en día se considera a la Andragogía como la disciplina que se ocupa de la educación y el aprendizaje de los jóvenes y adultos, a diferencia de la Pedagogía que se aplicó a la educación del niño y el adolescente.

El acto pedagógico, tradicionalmente visto, es formativo, se centra en la actividad del profesor, quien trasmite al niño $o$ al adolescente los contenidos que el sistema educativo les impone, siendo muy limitadas las posibilidades de cuestionar por sus escasas experiencias previas. Todo posee para ellos un carácter novedoso, su capacidad de asombro, si es bien canalizada, constituye una de las más poderosas motivaciones para aprender.

Por otro lado, el acto andragógico es un modelo que intenta tomar en cuenta esta situación de la enseñanza centrada en el profesor, y establece otro patrón consistiendo en la educación centrada en el alumno joven o adulto, teniendo en razones el tiempo vivido por ellos y en consecuencia, su experiencia previa. El profesor se convierte en facilitador, en vez de autoridad. Aunque estos métodos indican la participación activa de los jóvenes y adultos, el profesor - facilitador es necesario para proveer contexto social y seguridad emocional.

El acto andragógico será siempre voluntario, ya que el joven o adulto, aun siendo obligado a asistir o participar en clases, tiene la facultad y libertad para decidir sobre su educación e instrucción. En otras palabras, irá a las sesiones de clases, pero su mente estará en otra parte, si además no se considera su experiencia y no tiene oportunidad de participar, todo lo que se haga será en vano.

A diferencia de lo que ocurre con el niño, el adulto tiene una visión clara de la vida y una percepción de la escasez temporal, por lo que tiende a buscar contenidos prácticos, específicos y de utilidad inmediata, es decir, tiene que estar convencido para qué le servirá lo que se le enseña. Es, por lo tanto, selectivo (de un modo no necesariamente consciente) centrándose en todo aquello que sí cree poder dominar, descartando posibilidades de acuerdo a sus capacidades, intereses y limitaciones. No quiere perder su tiempo. En este enfoque el profesor es el facilitador de un proceso liberador que se basa en el intercambio de experiencias entre adultos y que tiene como propósito central ofrecer al adulto oportunidades de elaborar soluciones, liberadas del elevado costo del error. Queda en claro entonces, que son dos procesos diferentes que no debieran ser abordados del mismo modo, ni con las mismas técnicas.

Los autores de este trabajo consideran que el efecto multiplicador en la eficiencia del aprendizaje se logra evitando las debilidades de ambos modelos: el papel autoritario del profesor en situaciones pedagógicas y la dependencia excesiva en el conocimiento que tengan los educandos jóvenes y adultos en la situación andragógica y preservando los aspectos fuertes, es decir, el papel del profesor en proporcionar las situaciones y materiales necesarios sobre el tema a estudiar y la participación activa de los educandos jóvenes y adultos.

Casi es una metodología de educación y entrenamiento de adultos en la que métodos pedagógicos convencionales se adaptan a la participación activa de los educandos, sobre la base de su experiencia vivida, sus intereses y conocimientos previos, pero para que el éxito funcione, los profesionales que se desempeñan como docentes deben tener conocimiento de las posibilidades reales de los jóvenes y adultos con los cuales trabajan, haber reflexionado sobre ellos, quiénes son, qué saben, cómo se construyeron sus conocimientos, cómo leen sus experiencias, cuáles son sus motivos e intereses. Esta es una condición indispensable para promover el desarrollo de los procesos cognoscitivos y de las cualidades de la personalidad, dirigidas al saber hacer y saber convivir.

\section{¿Cómo debe ser el profesor de la nueva universidad cubana?}

En su libro Pedagogía de la autonomía, (Freire, P. 2018), pedagogo brasileño que dedicó gran parte de su vida a la enseñanza con personas jóvenes y adultas en diferentes contextos socioculturales, propone un perfil ideal para los educadores que se desempeñan con estos educandos, basado en las exigencias de la profesión de enseñar, tales como: rigor metodológico, respeto a los saberes, actitud crítica hacia el conocimiento, diálogo y rechazo de cualquier forma de discriminación. Y otras como: reflexión crítica sobre la práctica, reconocimiento de la asunción de la identidad cultural, respeto a la autonomía, buen juicio, 
humildad, tolerancia y lucha en defensa de los educadores, aprehensión de la realidad, alegría y esperanza, curiosidad y compromiso. El educador debe comprender que la educación es una forma de intervención en el mundo que implica libertad y autoridad.

Teniendo en cuenta que nuestro sistema social precisa de profesionales sustentados en la formación de una cultura general integral que se fundamenta en la consideración del hombre como un ser biopsicosocial individual, traducido esto en un dominio casi perfecto de los contenidos de su especialidad, contextualizados al entorno del territorio o la comunidad en la cual convive, para transformarla en bien de los objetivos y programas sociales, el perfil del profesional que se desempeña como docente en la nueva universidad cubana, va mucho más allá del perfil propuesto por Freire.

El profesional que se desempeña como docente en la nueva universidad cubana, además de lo planteado por Freire, debe caracterizarse por:

- Poseer una cultura política, fundamentada en el conocimiento y dominio de la Constitución de la República, del sistema electoral cubano, la aplicación del pensamiento revolucionario de los héroes y mártires de la patria, expresando su rechazo al capitalismo, al poder hegemónico del imperialismo yanqui y asumiendo una posición consciente para la defensa y conservación de las conquistas del socialismo cubano.

- Conocer la Historia de la Educación Superior en Cuba, así como los rasgos que la caracterizan y diferencian con el resto del mundo para poder fundamentar los actuales procesos de cambio que enfrenta esta enseñanza.

- Conocer la situación actual que tiene la Educación Superior en el mundo, las causas que la sustentan, así como las consecuencias que provocan al desarrollo de los países, a la paz y felicidad de los pueblos.

- Tener pleno dominio del plan de estudio y de los programas de las asignaturas que imparte, de modo que le permita ubicarse, ante los alumnos jóvenes y adultos con los que trabaja, como fuente de conocimientos, experiencias e informaciones.

- Estar dotado de habilidades comunicativas que le permitan acercarse al resto del claustro y de manera especial, a sus alumnos jóvenes y adultos, conocer sus motivaciones, intereses, inquietudes $\mathrm{y}$ conocimientos que, por muy difusos que sean, son el punto de partida para impulsar la construcción del nuevo conocimiento.

- Ser creativo para potenciar el interés de los jóvenes y adultos en todas sus esferas de actuación y propiciar un ambiente de trabajo que favorezca la empatía y el aprendizaje.

- Dominar a plenitud las funciones del profesor tutor, para poder ejercerse como puente vivo entre las actividades relacionadas con la vida académica, laboral, investigativa, familiar y social de sus alumnos.

\section{Ejes fundamentales en el proceso docente - educativo con personas jóvenes y adultas}

Teniendo en cuenta que las matrículas de la actual universidad cubana están dotadas de una composición heterogénea en cuanto a edades, procedencia social, conocimientos y ritmos de aprendizaje en sus alumnos, el proceso docente educativo que desarrolla el profesional que ejerce como docente en este nivel de enseñanza, debe estar fundamentado sobre la base de tres ejes principales que sostienen la interacción entre ambos participantes de dicho proceso, estos son:

1. Eje de la comunicación.

2. Eje de la emocionalidad.

3. Eje de formación.

\section{Eje de la Comunicación}

Desde la perspectiva que el proceso docente educativo, es de entrada, un acto comunicativo, el profesor de la nueva universidad cubana que se ha sensibilizado con su función, también será un observador de la calidad del proceso de comunicación entre él y sus discípulos. En este proceso de comunicación se deben cumplir con 
los requisitos básicos siguientes: claridad, fluidez y escucha activa.

- La claridad se manifiesta en que el profesional que se desempeña como docente en las condiciones de universalización, con matrículas compuestas por personas jóvenes y adultas de diferentes características, debe comprender de forma clara lo que sus alumnos le solicitan, debe corregirlos oportunamente cuando estos no expresen correctamente sus necesidades de aprendizaje. Una tarea ineludible del profesor de este nivel de enseñanza en las condiciones actuales de nuestro país, es su actividad constante de esclarecimiento de los mensajes de sus alumnos.

- La fluidez se manifiesta en que el profesional que se desempeña como docente en las condiciones de universalización, esté atento a observar los mensajes emitidos por sus alumnos, tratando de dar respuestas claras y convincentes en la medida de sus posibilidades. Las demandas de los alumnos, en ocasiones quedan fuera de toda posibilidad de respuesta por parte del profesor, aun así el profesor orienta a este para buscar un espacio que responda a sus inquietudes y necesidades de aprendizaje.

- En la escucha activa el profesional que se desempeña como docente en las condiciones de universalización, debe escuchar de modo integral los planteamientos de sus alumnos. Debe ser un excelente observador del lenguaje no verbal que se presenta en la interacción con los mismos. Es importante recordar que además de escuchar por el oído también se "escucha" por la observación y que se puede tener información muy relevante al observar movimientos, gestos, vestimenta, etc, que nos puede ser útil para propiciar la construcción del nuevo conocimiento.

\section{Eje de la Emocionalidad}

- En el caso del contexto, las condiciones y los retos que imponen el proceso de universalización de la Educación Superior cubana, las emociones juegan un papel fundamental, ya que con un estado emocional definido y adecuado, los alumnos se acercan al profesor, buscando apoyo, escucha, comprensión, ayuda y solución a sus problemas de aprendizaje. Recibe lo que el profesor ofrece como respuesta, también con cierta emocionalidad, abrigando deseos y expectativas propias para cada uno de ellos. El equilibrio emocional es básico en la función docente en lo correspondiente a la interacción en el proceso docente educativo, solo un buen equilibrio emocional en el profesor, permitirá un proceso efectivo.

\section{Eje de Formación}

- El profesional que se desempeña como docente en el proceso de universalización de la Educación Superior cubana, tiene una privilegiada oportunidad de aportar a la formación de sus alumnos una actitud abierta y sin perjuicios, una disposición a la ayuda y a la colaboración, la posibilidad de escuchar y respetar en la relación con sus alumnos, son ejemplos y posibilidades de aprendizaje cada momento en que se interactúa, este toma elementos de esa interacción que, difícilmente se aprenderán en clases durante una excelente exposición del profesor.

- La interacción en el proceso docente educativo, ofrece constantemente la oportunidad de aportar actitudes y valores a la formación de los alumnos, a través de sus vivencias.

¿Qué debe tener en cuenta el profesional que se desempeña como profesor en los centros universitarios municipales para tener éxitos en su trabajo?

Para el enriquecimiento de su trabajo y un óptimo desempeño de la labor docente educativa, es necesario que el profesor cubano que se desempeña en las condiciones del proceso de universalización, tenga en cuenta tres aspectos fundamentales:

1. La manera en que aprenden los jóvenes y adultos. 
2. Los requerimientos o requisitos para enseñar a los jóvenes y adultos.

3. El diagnóstico.

\section{La manera en que aprenden los jóvenes y adultos}

Una visión de la manera en que aprenden los jóvenes y adultos puede ser de gran ayuda para los profesionales que se desempeñan en las condiciones del proceso de universalización. Investigaciones realizadas han desechado la idea de que la capacidad de los adultos para aprender disminuye con la edad, lo que demuestra que pueden aprender a cualquier edad. Sobre la forma de aprender de los jóvenes y adultos se ha podido constatar que:

- Tienen que estar motivados para el aprendizaje, de no ser así, se resistirán cuando alguien simplemente les dice qué tienen que aprender. Se puede despertar su interés, o estimularlos, pero no forzarlos.

- Son prácticos, aprenderán solamente lo que creen que necesitan saber o quieren saber. Tampoco se satisfacen con asegurarles que eventualmente verán la importancia de lo que van a aprender. Esperan resultados a la primera sesión, por tal motivo, lo que se enseña tiene que ser adaptado a las necesidades, motivaciones e intereses de los participantes y no a las del profesor.

- Aprenden al hacer cosas, se olvidan dentro de un año del 50\% de lo que aprenden de manera pasiva, por lo que la retención de la información o habilidades es mucho mayor si existen oportunidades repetidas para practicar o usar lo que se está enseñando.

- La función de aprender se centra en problemas que sean realistas y que se ajusten a sus necesidades e intereses. Se puede enseñar principios y una serie de ilustraciones hipotéticas, pero las investigaciones demuestran que aprenden mejor haciendo este proceso al revés, es decir, trabajar con problemas reales, que saquen sus propias soluciones, y luego deduzcan los principios generales.

- Aprenden mejor en una situación informal.

- El uso de una mayor variedad de métodos para instruir es más efectivo porque se utilizan más vías sensoriales para hacer llegar el mensaje. Esto está basado en que el joven o adulto capta un $10 \%$ de lo que lee, un $20 \%$ de lo que escucha, un $30 \%$ de lo que ve, un $50 \%$ de lo que ve y escucha y un $80 \%$ de lo que escucha y hace.

- Se muestran impacientes con el formalismo de la educación, pero a la vez requieren de la autoevaluación de su aprendizaje, así que el profesor debe proporcionar el máximo de retroalimentación que pueda, sean elogios o críticas, siempre que se sea honesto y diplomático.

\section{Los requerimientos o requisitos para enseñar a los jóvenes y adultos}

El desempeño profesional de un profesor de personas jóvenes y adultas, como cualquier otra actividad humana, está sujeta a una serie de circunstancias y requerimientos. El grado de éxito dependerá de que el profesor reúna ciertos requisitos, siendo los siguientes:

- Que quiera hacer: Es condición vital que el profesor de jóvenes y adultos manifieste el deseo de compartir con sus alumnos sus conocimientos y sus experiencias, que goce con el desempeño de esta actividad.

- Que sepa lo que debe hacer: Es condición sabida que nadie podrá enseñar lo que no sabe. Nadie puede brindar lo que no tiene, por tanto, el grado de dominio de las asignaturas que imparte el profesor que se desempeña con jóvenes y adultos, debe ser mayor que lo que se espera que enseñe.

- Que sepa cómo hacerlo: El solo dominio que pueda tener, del contenido de las asignaturas que imparte, el profesor de jóvenes y adultos, no da la garantía de saber comunicarlo en términos de optimizar el aprendizaje. Un profesor puede ser un sabio en un tema determinado $y$ puede ser pésimo al enseñárselo a sus alumnos. El profesor deberá estar pendiente en todo momento de los mensajes no verbales del grupo, los cuales le brindan información sobre el grado en que se está realizando la comunicación.

- El saber cómo permitirá al profesor ser ágil en el uso y variación de las técnicas y métodos más eficaces para el logro de los objetivos académicos y formativos que se ha 
trazado, combinando Andragogía y Pedagogía, en busca de la eficacia y la eficiencia del proceso docente educativo, en correspondencia con las características de sus alumnos.

- Que sepa cómo aprenden los adultos: Los estudios relativos a la manera como aprenden los jóvenes y adultos le dará la posibilidad de adquirir conocimientos y habilidades en el manejo y uso de las técnicas y ayudas audiovisuales en el aprendizaje de estos educandos, las cuales auxilian al profesor de este nivel de enseñanza a reducir barreras y hacer que sea óptimo el aprendizaje de los participantes.

- Que sepa comunicarse: es importante recalcar que un docente que se desempeñe con matrículas de jóvenes y adultos, necesita saber comunicarse hábilmente con el grupo. $Y$ lo que es más importante: Trasmitir imágenes y enseñar a partir de la experiencia adquirida, más que enseñar conceptos. A las personas jóvenes y adultas les resulta difícil retener y comprender los conceptos en términos abstractos, en cambio las imágenes y el aprendizaje a partir de la solución de situaciones prácticas del contexto social e individual de los participantes, son contenidos que fácilmente retenemos, memorizamos y significamos.

- Que sea flexible: recordemos que la profesionalidad de un profesor y más de esta enseñanza, por el grado de complejidad que exige, se manifiesta en la preparación que realice para el desempeño de su actividad, de ninguna forma debe ser alguien que improvise, por tanto, debe dedicar tiempo a la preparación del contenido, así como a la metodología y la didáctica para su impartición. Sin embargo, algo muy peculiar en las condiciones que impone la universalización es que, en ocasiones, hay que realizar ajustes al programa o a la planificación previa que se tenía. Ello puede obedecer al nivel del grupo, a un interés auténtico del mismo en un determinado tema, etc. Pues el profesor debe ser consecuente y flexible para realizar dichos ajustes y así orientarse de manera más atinada y apegada a los requerimientos e intereses del grupo. Si ante una situación fuera de orden, alguien debe reaccionar con mesura, se espera que sea el profesor. Ser flexible no significa cambiar por cambiar.

\section{El diagnóstico}

La universalización de la Educación Superior en Cuba es un proceso de transformación, planificado, conducido, ejecutado y evaluado desde los propios agentes receptores del mismo, con el objetivo de desarrollar sus conocimientos y habilidades, satisfacer sus necesidades espirituales, generar poder y profundizar en su identidad y compromiso revolucionario.

La heterogeneidad de conocimientos que manifiestan las matrículas de la actual Educación Superior cubana, la diversidad de experiencias y expectativas, unido a la diversidad de motivos e intereses, exigen, para el logro de lo planteado anteriormente, la realización de un diagnóstico con características muy bien definidas.

¿Cómo debe ser entonces el diagnóstico de los alumnos en las actuales circunstancias del proceso de universalización en Cuba?

En casi todos los casos, el diagnóstico para las matrículas jóvenes y adultas que asisten a nuestras aulas se concibe como un proceso para conocer a los alumnos y no para que los jóvenes y adultos se reconozcan por sí solos. Es decir, pesa mucho la tesis de la objetividad y se descuida la subjetividad individual y colectiva, que pudiera garantizarnos el involucramiento y participación de los jóvenes y adultos en el proceso de enseñanza - aprendizaje.

Luego, el diagnóstico para este nivel de enseñanza en tales condiciones debe caracterizarse por ser:

1. Auto reflexivo.

2. Triangular.

3. Participativo.

\section{¿Qué significa que sea auto reflexivo?}

- En múltiples ocasiones el diagnóstico está dirigido a conocer a los jóvenes y adultos desde fuera, no se realiza una reflexión con ellos, que les permita reconocer su propia realidad. Los docentes participan suministrando 
información, pero además de eso es necesario que los profesionales que se desempeñan en este nivel de enseñanza propicien un intercambio con estos jóvenes y adultos que les permita reconocerse a sí mismo, cuáles son sus conocimientos, experiencias, expectativas, cuál es su lugar en el proceso de aprendizaje, etc. Esto ayudaría al comprometimiento del sujeto con su propio aprendizaje.

\section{¿Por qué triangular?}

- Porque tiene que estar dirigido a que estos alumnos jóvenes y adultos, por su experiencia vivida, sean capaces de encontrar sus propias incoherencias entre lo que piensan, hacen y lo que requiere su contexto social que hagan. Es decir, el diagnóstico tiene que propiciar que ellos reconozcan sus criterios valorativos, sus necesidades, sus intereses, sus creencias, sus valores, para reflexionar en la búsqueda de incoherencias entre todo eso y sus modos de actuación en el contexto social en que se desenvuelven y del cual forman parte, pero que a la vez deben saber que ese contexto social se debe a una historia, un modelo con su estructura y formas organizativas, con sus potencialidades $\mathrm{y}$ líderes sociales, que ellos deben respetar.

\section{¿Por qué participativo?}

- Porque los alumnos, sean jóvenes o adultos, tienen que ser partícipes del diagnóstico, deben comprometerse con las valoraciones y decisiones que en él se asumen, tienen que llegar a sentir que son ellos quienes lo realizan, que son sujetos activos de ese proceso.

- Una vez concluido el diagnóstico, el accionar del profesor debe estar dirigido al aprendizaje o crecimiento que pudieran tener los jóvenes y adultos, tanto de manera individual como colectiva, no solo en el tema objeto de estudio, sino en las maneras de investigar, en las formas de organizarse y potenciar sus fuerzas, en la adquisición de nuevos conocimientos y nuevas formas comunicativas, en la cohesión, sentido de pertenencia, en el fortalecimiento de las relaciones humanas y en la formación de nuevos valores.

- Es necesario que los claustros de la Educación Superior, que se desempeñan bajo las condiciones del proceso de universalización en Cuba, tenga bien claro que cuando se habla de aprendizaje de personas jóvenes y adultas, no se habla de un proceso anexo, escolarizado, sino de un aprendizaje que se produce sobre el desarrollo mismo de las tareas, con el acompañamiento del profesor, con la reflexión previa del grupo, con la valoración crítica de la actividad, con la consulta de bibliografía y el ejercicio de entrenamiento sobre la base de la experiencia, con la conciencia de aprender haciendo.

\section{Conclusiones}

El contenido expuesto en el presente trabajo se puede utilizar como material básico en la preparación inicial y permanente de los profesionales que han pasado a formar parte de los claustros de la Educación Superior, como consecuencia del proceso de universalización de la universidad cubana, ya que emerge a partir de un proceso de determinación de necesidades y garantiza, en su integración teórica, dar respuestas a las exigencias de este revolucionario proceso.

En el trabajo se han considerado las principales áreas de formación para el desempeño de las funciones del profesor cubano de la Educación Superior en condiciones de universalización y su integración tiene el objetivo de actualizarlo desde una dimensión teórica, metodológica y didáctica.

Los métodos de investigación utilizados en la realización del presente trabajo, le confieren seguridad teórica, metodológica y didáctica a la propuesta que hacemos, pues en su contextualización condicionan la consistencia interna, transferibilidad y aplicabilidad en el contexto de la actual universidad cubana. 


\section{Referencias}

Álvarez De Zayas, C. M, (1998). Fundamentos teóricos de la dirección del proceso de formación del profesional de perfil amplio. Santa Clara: Ediciones UCLVU.

Álvarez De Zayas, C. M, (1992). La escuela en la vida. La Habana: Editorial Pueblo y Educación.

Addine Fernández, F. (2004). Didáctica: teoría y práctica. La Habana: Ed. Pueblo y Educación.

Añorga Morales, J. (1995). Teoría de los sistemas de superación. La Habana: Conferencia dictada en el 1er Taller de educación de Avanzada, Ciencia y Técnica. Material impreso.

Beccaría, L.P. Aspectos pedagógicos de la capacitación de adultos. Recuperado en http://www.nps.gov/oia/Section3.pdf

Covelo, M. C. (206). Educación para Adultos. Recuperado en http://www.capitannemo.com.ar/educacion_para_adultos.htm

Dueñas, M. Á. (2001). Cambio social y formación del profesorado. Madrid: Tarbiya.

Freire, P. (2018). Pedagogía de la autonomía. Recuperado en http://sigloxxieditores.com.mx

García Otero, J. (2002). Un nuevo reto en la formación del personal docente: la municipalización de la universidad pedagógica. La Habana: Ed. Ciencia y Técnica. 